\title{
On Nonlinear Fractional Sum-Difference Equations via Fractional Sum Boundary Conditions Involving Different Orders
}

\author{
Saowaluk Chasreechai, Chanakarn Kiataramkul, and Thanin Sitthiwirattham
}

Nonlinear Dynamic Analysis Research Center, Department of Mathematics, Faculty of Applied Science, King Mongkut's University of Technology North Bangkok, Bangkok 10800, Thailand

Correspondence should be addressed to Thanin Sitthiwirattham; thanin.s@sci.kmutnb.ac.th

Received 13 July 2015; Revised 3 September 2015; Accepted 6 September 2015

Academic Editor: Sotiris K. Ntouyas

Copyright (C) 2015 Saowaluk Chasreechai et al. This is an open access article distributed under the Creative Commons Attribution License, which permits unrestricted use, distribution, and reproduction in any medium, provided the original work is properly cited.

We study existence and uniqueness results for Caputo fractional sum-difference equations with fractional sum boundary value conditions, by using the Banach contraction principle and Schaefer's fixed point theorem. Our problem contains different numbers of order in fractional difference and fractional sums. Finally, we present some examples to show the importance of these results.

\section{Introduction}

In this paper we consider a Caputo fractional sum-difference equation with nonlocal fractional sum boundary value conditions of the form

$$
\begin{aligned}
& \Delta_{C}^{\alpha} u(t) \\
& \quad=f\left(t+\alpha-1, u(t+\alpha-1),\left(\Psi^{\beta} u\right)(t+\alpha-2)\right), \\
& \quad t \in \mathbb{N}_{0, T}:=\{0,1, \ldots, T\}, \\
& \begin{array}{l}
u(\alpha-2)=y(u), \\
u(T+\alpha)=\Delta^{-\gamma} g(T+\alpha+\gamma-3) u(T+\alpha+\gamma-3),
\end{array}
\end{aligned}
$$

where $1<\alpha \leq 2,0<\beta \leq 1,2<\gamma \leq 3$, and $\Delta_{C}^{\alpha}$ is the Caputo fractional difference operator of order $\alpha$. For $U \subseteq \mathbb{R}$, $g \in C\left(\mathbb{N}_{\alpha-2, T+\alpha}, \mathbb{R}^{+} \cap U\right)$ and $f \in C\left(\mathbb{N}_{\alpha-2, T+\alpha} \times U \times U, U\right)$ are given functions and $y: C\left(\mathbb{N}_{\alpha-2, T+\alpha}, U\right) \rightarrow U$ is a given functional, and for $\varphi: \mathbb{N}_{\alpha-2, T+\alpha} \times \mathbb{N}_{\alpha-2, T+\alpha} \rightarrow[0, \infty)$,

$$
\begin{aligned}
& \left(\Psi^{\beta} u\right)(t):=\left[\Delta^{-\beta} \varphi u\right](t+\beta) \\
& =\frac{1}{\Gamma(\beta)} \sum_{s=\alpha-\beta-2}^{t-\beta}(t-\sigma(s)) \frac{\beta-1}{-} \varphi(t, s+\beta) u(s+\beta) .
\end{aligned}
$$

Mathematicians have employed this fractional calculus in recent years to model and solve various applied problems.
In particular, fractional calculus is a powerful tool for the processes which appear in nature, for example, biology, ecology, and other areas, and can be found in $[1,2]$ and the references therein. The continuous fractional calculus has received increasing attention within the last ten years or so, and the theory of fractional differential equations has been a new important mathematical branch due to its extensive applications in various fields of science, such as physics, mechanics, chemistry, and engineering. Although the discrete fractional calculus has seen slower progress, within the recent several years, a lot of papers have appeared, which has helped to build up some of the basic theory of this area; see [3-17] and references cited therein.

At present, there is a development of boundary value problems for fractional difference equations which shows an operation of the investigative function. The study may also have another function which is related to the one we are interested in. These creations are incorporating with nonlocal conditions which are both extensive and more complex, for instance.

Agarwal et al. [3] investigated the existence of solutions for two fractional boundary value problems:

$$
\begin{aligned}
& \Delta_{\mu-2}^{\mu} x(t) \\
& \quad=g(t+\mu-1, x(t+\mu-1), \Delta x(t+\mu-1)), \\
& \quad t \in \mathbb{N}_{0, b+2},
\end{aligned}
$$




$$
\begin{aligned}
& x(\mu-2)=0, \\
& x(\mu+b+1)=\sum_{k=\mu-1}^{\alpha} x(k),
\end{aligned}
$$

where $1<\mu \leq 2$ and $g \in C\left(\mathbb{N}_{\mu-1, \mu+b+1} \times \mathbb{R} \times \mathbb{R}, \mathbb{R}\right)$ is a given function, and

$$
\begin{aligned}
\Delta_{\mu-3}^{\mu} x(t) & =g(t+\mu-2, x(t+\mu-2)), \quad t \in \mathbb{N}_{0, b+3}, \\
x(\mu-3) & =x(\mu+b+1)=0, \\
x(\alpha) & =\sum_{k=\gamma}^{\beta} x(k),
\end{aligned}
$$

where $2<\mu \leq 3, \alpha, \beta, \gamma \in \mathbb{N}_{\mu-2, \mu+b}$, and $g \in C\left(\mathbb{N}_{\mu-2, \mu+b+1} \times\right.$ $\mathbb{R} \times \mathbb{R}, \mathbb{R})$ is a given function.

Kang et al. [5] obtained sufficient conditions for the existence of positive solutions for a nonlocal boundary value problem

$$
\begin{aligned}
& -\Delta^{\mu} y(t)=\lambda h(t+\mu-1) f(y(t+\mu-1)), \\
& y(\mu-2)=\Psi(y), \\
& y(\mu+b)=\Phi(y),
\end{aligned}
$$

where $1<\mu \leq 2, f \in C([0, \infty),[0, \infty)), h \in$ $C\left(\mathbb{N}_{\mu-1, \mu+b-1},[0, \infty)\right)$ are given functions and $\Psi, \Phi: \mathbb{R}^{b+3} \rightarrow$ $\mathbb{R}$ are given functionals.

Sitthiwirattham [17] examined a Caputo fractional sum boundary value problem with a $p$-Laplacian of the form

$$
\begin{aligned}
& \Delta_{C}^{\alpha}\left[\phi_{p}\left(\Delta_{C}^{\beta} x\right)\right](t) \\
& \quad=f(t+\alpha+\beta-1, x(t+\alpha+\beta-1)), \quad t \in \mathbb{N}_{0, T}, \\
& \Delta_{C}^{\beta} x(\alpha-1)=0, \\
& x(\alpha+\beta+T)=\rho \Delta^{-\gamma} x(\eta+\gamma),
\end{aligned}
$$

where $0<\alpha, \beta \leq 1,1<\alpha+\beta \leq 2,0<\gamma \leq 1$, $\eta \in \mathbb{N}_{\alpha+\beta-1, \alpha+\beta+T-1}, \rho$ is a constant, $f: \mathbb{N}_{\alpha+\beta-2, \alpha+\beta+T} \times \mathbb{R} \rightarrow \mathbb{R}$ is a continuous function, and $\phi_{p}$ is the $p$-Laplacian operator.

The plan of this paper is as follows. In Section 2 we recall some definitions and basic lemmas. Also we derive a representation for the solution to (1) by converting the problem to an equivalent summation equation. In Section 3, using this representation, we prove existence and uniqueness of the solutions of boundary value problem (1) by the help of the Banach fixed point theorem and Schaefer's fixed point theorem. Some illustrative examples are presented in Section 4.

\section{Preliminaries}

In the following, there are notations, definitions, and lemmas which are used in the main results.

Definition 1. One defines the generalized falling function by $t^{\underline{\alpha}}:=\Gamma(t+1) / \Gamma(t+1-\alpha)$, for any $t$ and $\alpha$ for which the right-hand side is defined. If $t+1-\alpha$ is a pole of Gamma function and $t+1$ is not a pole, then $t^{\underline{\alpha}}=0$.

Lemma 2 (see [13]). Assume that the following factorial functions are well defined:

(i) $(t-\mu) t^{\mu}=t^{\mu+1}$, where $\mu \in \mathbb{R}$.

(ii) If $t \leq r$, then $t^{\underline{\alpha}} \leq r^{\underline{\alpha}}$ for any $\alpha>0$.

(iii) $t \stackrel{\alpha+\beta}{\underline{\beta}}=(t-\beta)^{\underline{\alpha}} t \underline{\beta}$.

Definition 3. For $\alpha>0$ and $f$ defined on $\mathbb{N}_{a}:=\{a, a+1, \ldots\}$, the $\alpha$-order fractional sum of $f$ is defined by

$$
\Delta^{-\alpha} f(t):=\frac{1}{\Gamma(\alpha)} \sum_{s=a}^{t-\alpha}(t-\sigma(s))^{\frac{\alpha-1}{}} f(s),
$$

where $t \in \mathbb{N}_{a+\alpha}$ and $\sigma(s)=s+1$.

Definition 4. For $\alpha>0$ and $f$ defined on $\mathbb{N}_{a}$, the $\alpha$-order Caputo fractional difference of $f$ is defined by

$$
\begin{aligned}
\Delta_{C}^{\alpha} f(t) & :=\Delta^{-(N-\alpha)} \Delta^{N} f(t) \\
& =\frac{1}{\Gamma(N-\alpha)} \sum_{s=a}^{t-(N-\alpha)}(t-\sigma(s))^{\frac{N-\alpha-1}{}} \Delta^{N} f(s),
\end{aligned}
$$

where $t \in \mathbb{N}_{a+N-\alpha}$ and $N \in \mathbb{N}$ is chosen so that $0 \leq N-1<$ $\alpha<N$. If $\alpha=N$, then $\Delta_{C}^{\alpha} f(t)=\Delta^{N} f(t)$.

Lemma 5 (see [9]). Assume that $\alpha>0$ and $0 \leq N-1<\alpha \leq$ $N$. Then

$$
\begin{aligned}
\Delta^{-\alpha} \Delta_{C}^{\alpha} y(t)= & y(t)+C_{0}+C_{1} t^{\underline{1}}+C_{2} t^{2}+\cdots \\
& +C_{N-1} t^{\frac{N-1}{2}}
\end{aligned}
$$

for some $C_{i} \in \mathbb{R}, 0 \leq i \leq N-1$.

The following lemma deals with linear variant of boundary value problem (1) and gives a representation of the solution.

Lemma 6. Let $1<\alpha \leq 2,2<\gamma \leq 3, y: C\left(\mathbb{N}_{\alpha-2, T+\alpha}, U\right) \rightarrow$ $U$, and $h \in C\left(\mathbb{N}_{\alpha-1, T+\alpha-1}, U\right)$ be given. Then the problem

$$
\begin{aligned}
\Delta_{C}^{\alpha} u(t) & =h(t+\alpha-1), \quad t \in \mathbb{N}_{0, T}, \\
u(\alpha-2) & =y(u), \\
u(T+\alpha) & =\Delta^{-\gamma} g(T+\alpha+\gamma-3) u(T+\alpha+\gamma-3),
\end{aligned}
$$

has the unique solution

$u(t)$

$$
\begin{aligned}
= & \left(1-\frac{t^{\underline{1}}}{T+\alpha}\right) y(u)+\frac{t^{\underline{1}}}{T+\alpha} A(u) \\
& -\frac{t^{\underline{1}}}{(T+\alpha) \Gamma(\alpha)} \sum_{s=\alpha-1}^{T+\alpha-1}(T+2 \alpha-1-\sigma(s))^{\frac{\alpha-1}{}} h(s) \\
& +\frac{1}{\Gamma(\alpha)} \sum_{s=\alpha-1}^{t-1}(t+\alpha-1-\sigma(s))^{\frac{\alpha-1}{}} h(s),
\end{aligned}
$$


where

$$
\begin{aligned}
& A(u)=-\frac{y(u) \sum_{s=\alpha-2}^{T+\alpha-3} g(s)(T+\alpha+\gamma-3+\sigma(s))^{\frac{\gamma-1}{-}}\left(1-s^{\underline{1}} /(T+\alpha)\right)}{(1 /(T+\alpha)) \sum_{s=\alpha-2}^{T+\alpha-3} s^{-1} g(s)(T+\alpha+\gamma-3+\sigma(s))^{\frac{\gamma-1}{}}-\Gamma(\gamma)} \\
& -\frac{(1 / \Gamma(\alpha)) \sum_{s=\alpha}^{T+\alpha-3} \sum_{\xi=\alpha-1}^{s-1} g(s)(T+\alpha+\gamma-3+\sigma(s))^{\gamma-1}(s+\alpha-1-\sigma(\xi))^{\frac{\alpha-1}{2}} h(\xi)}{(1 /(T+\alpha)) \sum_{s=\alpha-2}^{T+\alpha-3} s-\underline{1} g(s)(T+\alpha+\gamma-3+\sigma(s))^{\frac{\gamma-1}{-}}-\Gamma(\gamma)} \\
& +\frac{(1 / \Gamma(\alpha)) \sum_{s=\alpha}^{T+\alpha-3} \sum_{\xi=\alpha-1}^{T+\alpha-1} g(s) s^{\underline{1}}(T+\alpha+\gamma-3+\sigma(s))^{\gamma^{\gamma-1}}(T+2 \alpha-1-\sigma(\xi))^{\alpha-1} h(\xi)}{\sum_{s=\alpha-2}^{T+\alpha-3} s^{1} g(s)(T+\alpha+\gamma-3+\sigma(s))^{\frac{\gamma-1}{-}}-\Gamma(\gamma)} .
\end{aligned}
$$

Proof. Using Lemma 5, a general solution for (10) can be written in the form

$$
\begin{aligned}
u(t)= & C_{0}+C_{1} t^{\underline{1}} \\
& +\frac{1}{\Gamma(\alpha)} \sum_{s=0}^{t-\alpha}(t-\sigma(s))^{\frac{\alpha-1}{2}} h(s+\alpha-1),
\end{aligned}
$$

for $t \in \mathbb{N}_{\alpha-2, \alpha+T}$. Applying the first boundary condition of (10) implies

$$
C_{0}=y(u) .
$$

So,

$$
\begin{aligned}
u(t)= & y(u)+C_{1} t^{\underline{1}} \\
& +\frac{1}{\Gamma(\alpha)} \sum_{s=0}^{t-\alpha}(t-\sigma(s))^{\frac{\alpha-1}{}} h(s+\alpha-1) .
\end{aligned}
$$

The second condition of (10) implies

$$
\begin{aligned}
u(T+\alpha) & \\
= & y(u)+C_{1}(T+\alpha) \\
& +\frac{1}{\Gamma(\alpha)} \sum_{s=0}^{T}(T+\alpha-\sigma(s))^{\frac{\alpha-1}{2}} h(s+\alpha-1) \\
= & \frac{1}{\Gamma(\gamma)} \sum_{s=\alpha-2}^{T+\alpha-3}(T+\alpha+\gamma-3-\sigma(s))^{\frac{\gamma-1}{}} g(s) u(s) .
\end{aligned}
$$

A constant $C_{1}$ can be obtained by solving the above equation, so

$$
\begin{aligned}
C_{1}= & \frac{1}{(T+\alpha) \Gamma(\gamma)} \\
& \cdot \sum_{s=\alpha-2}^{T+\alpha-3}(T+\alpha+\gamma-3-\sigma(s))^{\frac{\gamma-1}{}} g(s) u(s)
\end{aligned}
$$

$$
\begin{aligned}
- & \frac{y(u)}{T+\alpha}-\frac{1}{(T+\alpha) \Gamma(\alpha)} \\
& \cdot \sum_{s=0}^{T}(T+\alpha-\sigma(s))^{\alpha-1} h(s+\alpha-1) .
\end{aligned}
$$

Substituting a constant $C_{1}$ into (15), we get

$$
\begin{gathered}
u(t)=\left(1-\frac{t^{1}}{T+\alpha}\right) y(u)+\frac{t^{\underline{1}}}{T+\alpha}\left[\frac{1}{\Gamma(\gamma)}\right. \\
\cdot \sum_{s=\alpha-2}^{T+\alpha-3}(T+\alpha+\gamma-3-\sigma(s))^{\frac{\gamma-1}{}} g(s) u(s) \\
\left.-\frac{1}{\Gamma(\alpha)} \sum_{s=\alpha-1}^{T+\alpha-1}(T+2 \alpha-1-\sigma(s))^{\frac{\alpha-1}{2}} h(s)\right] \\
+\frac{1}{\Gamma(\alpha)} \sum_{s=\alpha-1}^{t-1}(t+\alpha-1-\sigma(s))^{\frac{\alpha-1}{h}} h(s) .
\end{gathered}
$$

Let $A(u)=(1 / \Gamma(\gamma)) \sum_{s=\alpha-2}^{T+\alpha-3}(T+\alpha+\gamma-3-\sigma(s)) \frac{\gamma-1}{=} g(s) u(s)$. Then

$$
\begin{gathered}
A(u)=\frac{1}{\Gamma(\gamma)} \sum_{s=\alpha-2}^{T+\alpha-3} g(s)(T+\alpha+\gamma-3-\sigma(s))^{\frac{\gamma-1}{-1}} \\
\quad\left\{\left(1-\frac{s^{-}}{T+\alpha}\right) y(u)+\frac{s^{\underline{1}}}{T+\alpha}[A(u)\right. \\
\left.-\frac{1}{\Gamma(\alpha)} \sum_{\xi=\alpha-1}^{T+\alpha-1}(T+2 \alpha-1-\sigma(\xi))^{\frac{\alpha-1}{2}} h(\xi)\right] \\
\left.+\frac{1}{\Gamma(\alpha)} \sum_{\xi=\alpha-1}^{s-1}(s+\alpha-1-\sigma(\xi))^{\frac{\alpha-1}{2}} h(\xi)\right\},
\end{gathered}
$$

we simplify (19) becomes (12).

Substituting $A(u)$ into (18), we obtain (11). 


\section{Main Results}

Now we are in a position to establish the main results. First, we transform boundary value problem (1) into a fixed point problem.

For $U \subseteq \mathbb{R}$, let $(U,\|\cdot\|)$ be a Banach space and let $\mathscr{C}=$ $C\left(\mathbb{N}_{\alpha-2, T+\alpha}, U\right)$ denote the Banach space of all continuous functions from $\mathbb{N}_{\alpha-2, T+\alpha} \rightarrow U$ endowed with a topology of uniform convergence with the norm denoted by $\|\cdot\|_{\mathscr{C}}$. For this purpose, we consider the operator $F: \mathscr{C} \rightarrow \mathscr{C}$ by

$$
(F u)(t)=\left(1-\frac{t^{\underline{1}}}{T+\alpha}\right) y(u)+\frac{t^{\underline{1}}}{T+\alpha} A(u)
$$

$$
\begin{aligned}
& -\frac{t^{\underline{1}}}{(T+\alpha) \Gamma(\alpha)} \sum_{s=\alpha-1}^{T+\alpha-1}(T+2 \alpha-1-\sigma(s))^{\frac{\alpha-1}{}} \\
& \cdot f\left(s, u(s),\left(\Psi^{\beta} u\right)(s-1)\right)+\frac{1}{\Gamma(\alpha)} \\
& \cdot \sum_{s=\alpha-1}^{t-1}(t+\alpha-1-\sigma(s))^{\frac{\alpha-1}{}} \\
& \cdot f\left(s, u(s),\left(\Psi^{\beta} u\right)(s-1)\right),
\end{aligned}
$$

where

$$
\begin{aligned}
& A(u)=\frac{1}{(1 /(T+\alpha)) \sum_{s=\alpha-2}^{T+\alpha-3} s-\underline{1} g(s)(T+\alpha+\gamma-3-\sigma(s)) \frac{\gamma-1}{-\Gamma(\gamma)}}\left[-y(u) \sum_{s=\alpha-2}^{T+\alpha-3} g(s)(T+\alpha+\gamma-3-\sigma(s)) \frac{\gamma-1}{(1}\right. \\
& \left.-\frac{s^{\underline{1}}}{T+\alpha}\right)-\frac{1}{\Gamma(\alpha)} \sum_{s=\alpha}^{T+\alpha-3} \sum_{\xi=\alpha-1}^{s-1} g(s)(T+\alpha+\gamma-3-\sigma(s)) \frac{\gamma-1}{(s+\alpha-1-\sigma(\xi))^{\alpha-1}} f\left(\xi, u(\xi),\left(\Psi^{\beta} u\right)(\xi-1)\right)
\end{aligned}
$$

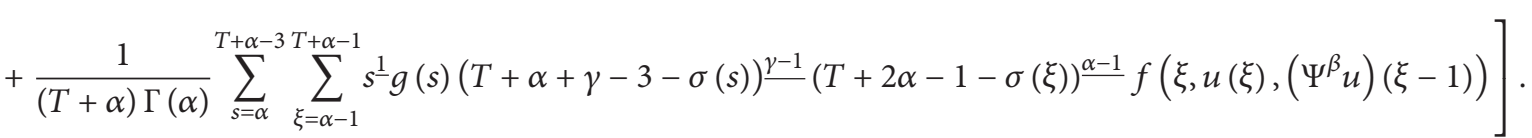

It is easy to see that problem (1) has solutions if and only if operator $F$ has fixed points.

Theorem 7. Assume that $f: \mathbb{N}_{\alpha-2, T+\alpha} \times U \times U \rightarrow U$ is continuous and maps bounded subsets of $\mathbb{N}_{\alpha-2, T+\alpha} \times U \times U$ into relatively compact subsets of $U, \varphi: \mathbb{N}_{\alpha-2, T+\alpha} \times \mathbb{N}_{\alpha-2, T+\alpha} \rightarrow$ $[0, \infty)$ is continuous with $\varphi_{0}=\max \{\varphi(t-1, s):(t, s) \in$ $\left.\mathbb{N}_{\alpha-2, T+\alpha} \times \mathbb{N}_{\alpha-2, T+\alpha}\right\}$, and $y: \mathscr{C} \rightarrow U$ is a given functional. In addition, suppose the following:

$\left(H_{1}\right)$ There exist constants $\tau_{1}, \tau_{2}>0$ such that for each $t \in \mathbb{N}_{\alpha-2, \alpha+T}$ and $u, v \in \mathscr{C}$

$$
\begin{aligned}
& \mid f\left(t, u(t),\left(\Psi^{\beta} u\right)(t-1)\right) \\
& \quad-f\left(t, v(t),\left(\Psi^{\beta} v\right)(t-1)\right)\left|\leq \tau_{1}\right| u-v \mid \\
& \quad+\tau_{2}\left|\left(\Psi^{\beta} u\right)-\left(\Psi^{\beta} v\right)\right| .
\end{aligned}
$$

$$
\begin{aligned}
& \Omega=2+\frac{K[\gamma(T+2)+3] \Gamma(T+\gamma)}{K[T+\alpha-\gamma(2-\alpha)-3]-(T+\alpha) \Gamma(\gamma+2) \Gamma(T)}, \\
& \Lambda=\frac{K \Gamma(T+\gamma-2)}{T \Gamma(\alpha+1) \Gamma(T-2)[K[T+\alpha-\gamma(2-\alpha)-3]-(T+\alpha) \Gamma(\gamma+2) \Gamma(T)]}[(\gamma+1) T(T-1)(T-2)(T+\alpha-2)
\end{aligned}
$$

$\left(H_{2}\right)$ There exists a constant $\mu>0$ such that for each $u, v \in \mathscr{C}$

$$
|y(u)-y(v)| \leq \mu\|u-v\|_{\mathscr{C}} .
$$

$\left(H_{3}\right)$ For each $t \in \mathbb{N}_{\alpha-2, \alpha+T}$

$$
\begin{aligned}
& 0<g(t)<K, \\
& K[T+\alpha-\gamma(2-\alpha)-3]-(T+\alpha) \Gamma(\gamma+2) \Gamma(T) \\
& \quad>0 .
\end{aligned}
$$

$\left(H_{4}\right)$ Consider $\Theta:=\mu \Omega+\Lambda\left(\tau_{1}+\tau_{2}\left(\varphi_{0}(T+\beta+2)-\beta\right.\right.$ 1))) $<1$,

where 


$$
+[T+\alpha(\alpha+\gamma)-3] \Gamma(T+\alpha)]+\frac{2 \Gamma(T+\alpha+1)}{\Gamma(\alpha+1) \Gamma(T+1)} .
$$

Then problem (1) has a unique solution on $\mathbb{N}_{\alpha-2, \alpha+T}$.
Proof. We will show that $F$ is a contraction. For any $u, v \in \mathscr{C}$ and for each $t \in \mathbb{N}_{\alpha-2, \alpha+T}$, we have

$$
\begin{aligned}
& |(F u)(t)-(F v)(t)| \leq\left|1-\frac{t^{\underline{1}}}{T+\alpha}\right||y(u)-y(v)|+\frac{t^{\underline{1}}}{T+\alpha}|A(u)-A(v)|+\frac{t^{\underline{1}}}{(T+\alpha) \Gamma(\alpha)} \sum_{s=\alpha-1}^{T+\alpha-1}(T-1-\sigma(s))^{\alpha-1} \\
& \cdot\left|f\left(s, u(s),\left(\Psi^{\beta} u\right)(s)\right)-f\left(s, v(s),\left(\Psi^{\beta} v\right)(s-1)\right)\right|+\frac{1}{\Gamma(\alpha)} \sum_{s=0}^{t-\alpha}(t-\sigma(s))^{\alpha-1} \mid f\left(s, u(s),\left(\Psi^{\beta} u\right)(s)\right) \\
& -f\left(s, v(s),\left(\Psi^{\beta} v\right)(s-1)\right) \mid<\mu\|u-v\|_{\mathscr{C}}\left[1+\frac{t^{1}}{T+\alpha}\right] \\
& +\frac{t^{\underline{1}}}{\left|\sum_{s=\alpha-2}^{T+\alpha-3} s^{1} g(s)(T+\alpha+\gamma-3-\sigma(s))^{\frac{\gamma-1}{-}}-(T+\alpha) \Gamma(\gamma)\right|}\left[\mu\|u-v\|_{\mathscr{C}} \sum_{s=\alpha-2}^{T+\alpha-3} g(s)(T+\alpha+\gamma-3-\sigma(s)) \frac{\gamma-1}{}(1\right. \\
& \left.-\frac{s^{\underline{1}}}{T+\alpha}\right)+\frac{\left[\left(\tau_{1}+\tau_{2}\left(\varphi_{0}(T+\beta+2)^{-\beta} / \Gamma(\beta+1)\right)\right)\|u-v\|_{\mathscr{C}}\right]}{\Gamma(\alpha)} \sum_{s=\alpha}^{T+\alpha-3} \sum_{\xi=\alpha-1}^{s-1} g(s)(T+\alpha+\gamma-3-\sigma(s)) \frac{\gamma-1}{(s+\alpha-1} \\
& -\sigma(\xi))^{\frac{\alpha-1}{2}}+\frac{\left[\left(\tau_{1}+\tau_{2}\left(\varphi_{0}(T+\beta+2)^{-\beta} / \Gamma(\beta+1)\right)\right)\|u-v\|_{\mathscr{C}}\right]}{(T+\alpha) \Gamma(\alpha)} \sum_{s=\alpha}^{T+\alpha-3} \sum_{\xi=\alpha-1}^{T+\alpha-1} s^{\underline{1}} g(s)(T+\alpha+\gamma-3-\sigma(s)) \frac{\gamma-1}{(T+2 \alpha} \\
& \left.-1-\sigma(\xi))^{\frac{\alpha-1}{2}}\right]+\frac{t^{\underline{1}}\left[\left(\tau_{1}+\tau_{2}\left(\varphi_{0}(T+\beta+2)^{-\beta} / \Gamma(\beta+1)\right)\right)\|u-v\|_{\mathscr{C}}\right]}{(T+\alpha) \Gamma(\alpha)} \sum_{s=\alpha-1}^{T+\alpha-1}(T+2 \alpha-1-\sigma(s))^{\frac{\alpha-1}{}} \\
& +\frac{\left[\left(\tau_{1}+\tau_{2}\left(\varphi_{0}(T+\beta+2)^{-\beta} / \Gamma(\beta+1)\right)\right)\|u-v\|_{\mathscr{C}}\right]}{\Gamma(\alpha)} \sum_{s=\alpha-1}^{t-1}(t+\alpha-1-\sigma(s))^{\frac{\alpha-1}{2}}<2 \mu\|u-v\|_{\mathscr{C}} \\
& +\frac{\mu\|u-v\|_{\mathscr{C}}(2 K \Gamma(T+\gamma+3) / \gamma \Gamma(T+3))}{K[T+\alpha-\gamma(2-\alpha)-3] / \gamma(\gamma+1) \Gamma(T)-(T+\alpha) \Gamma(\gamma)}+\left(\left(\tau_{1}+\tau_{2} \frac{\varphi_{0}(T+\beta+2) \underline{\beta}}{\Gamma(\beta+1)}\right)\|u-v\|_{\mathscr{C}}\right. \\
& \left.\cdot\left[\frac{K[\gamma(T+2)+3] \Gamma(T+\gamma)}{\gamma(\gamma+1) \Gamma(T)}+\frac{K \Gamma(T+\alpha(\alpha+\gamma)-3) \Gamma(T+\alpha) \Gamma(T+\gamma-2)}{\gamma(\gamma+1) \Gamma(\alpha+1) \Gamma(T-2) \Gamma(T+1)}\right]\right) \cdot\left(\frac{K[T+\alpha-\gamma(2-\alpha)-3]}{\gamma(\gamma+1) \Gamma(T)}\right. \\
& -(T+\alpha) \Gamma(\gamma))^{-1}+\frac{2\left(\tau_{1}+\tau_{2}\left(\varphi_{0}(T+\beta+2) \frac{\beta}{-} / \Gamma(\beta+1)\right)\right)\|u-v\|_{\mathscr{C}} \Gamma(T+\alpha+1)}{\Gamma(\alpha+1) \Gamma(T+1)}=\mu\|u-v\|_{\mathscr{C}}\{2 \\
& \left.+\frac{K[\gamma(T+2)+3] \Gamma(T+\gamma)}{K[T+\alpha-\gamma(2-\alpha)-3]-(T+\alpha) \Gamma(\gamma) \Gamma(T)}\right\}+\left(\left(\tau_{1}+\tau_{2} \frac{\varphi_{0}(T+\beta+2)^{-}}{\Gamma(\beta+1)}\right)\|u-v\|_{\mathscr{C}}\right) \\
& \text {. }\left\{\frac{K \Gamma(T+\gamma-2)}{T \Gamma(\alpha+1) \Gamma(T-2)[K[T+\alpha-\gamma(2-\alpha)-3]-(T+\alpha) \Gamma(\gamma+2) \Gamma(T)]}[(\gamma+1) T(T-1)(T-2)(T+\alpha-2)\right. \\
& \left.+[T+\alpha(\alpha+\gamma)-3] \Gamma(T+\alpha)]+\frac{2 \Gamma(T+\alpha+1)}{\Gamma(\alpha+1) \Gamma(T+1)}\right\}=\|u-v\|_{\mathscr{C}}\left\{\mu \Omega+\Lambda\left(\tau_{1}+\tau_{2} \frac{\varphi_{0}(T+\beta+2)^{\underline{\beta}}}{\Gamma(\beta+1)}\right)\right\} \\
& =\|u-v\|_{\mathscr{C}} \Theta \leq\|u-v\|_{\mathscr{C}} .
\end{aligned}
$$


Consequently, $F$ is a contraction. Therefore, by the Banach fixed point theorem, we get that $F$ has a fixed point which is a unique solution of problem (1) on $t \in \mathbb{N}_{\alpha-2, \alpha+T}$.

The following result is based on Schaefer's fixed point theorem.

Theorem 8 (Arzelá-Ascoli Theorem (see [18])). A set of function in $C[a, b]$ with the sup norm is relatively compact if and only if it is uniformly bounded and equicontinuous on $[a, b]$.

Theorem 9 (see [18]). If a set is closed and relatively compact then it is compact.

Theorem 10 (Schaefer's fixed point theorem (see [19])). Assume that $X$ is a Banach space and that $T: X \rightarrow X$ is continuous compact mapping. Moreover assume that the set

$$
\bigcup_{0 \leq \lambda \leq 1}\{x \in X: x=\lambda T(x)\}
$$

is bounded. Then T has a fixed point.

Theorem 11. Assume that $f: \mathbb{N}_{\alpha-2, T+\alpha} \times U \times U \rightarrow U$ is continuous and maps bounded subsets of $\mathbb{N}_{\alpha-2, T+\alpha} \times U \times U$ into relatively compact subsets of $U$ and $y: \mathscr{C} \rightarrow U$ is a given functional. In addition, suppose that $\left(\mathrm{H}_{3}\right)$ holds, and suppose the following:

$\left(H_{5}\right)$ There exists a constant $L_{1}>0$ such that for each $t \in \mathbb{N}_{\alpha-2, \alpha+T}$ and $u \in \mathscr{C}$

$$
\left|f\left(t, u(t),\left(\Psi^{\beta} u\right)(t-1)\right)\right| \leq L_{1} .
$$

$\left(H_{6}\right)$ There exists a constant $L_{2}>0$ such that for each $u \in \mathscr{C}$

$$
|y(u)| \leq L_{2}
$$

Then problem (1) has at least one solution on $\mathbb{N}_{\alpha-2, \alpha+T}$.

Proof. We will use Schaefer's fixed point theorem to prove this result. Let $F$ be the operator defined in (20). It is clear that $F: \mathscr{C} \rightarrow \mathscr{C}$ is completely continuous. So, it remains to show that the set

$$
\mathscr{E}=\{u \in \mathscr{C}: u=\lambda F u \text { for some } 0<\lambda<1\}
$$

is bounded.

Let $u \in \mathscr{E}$; then $u(t)=\lambda(F u)(t)$ for some $0<\lambda<1$. Thus, for each $t \in \mathbb{N}_{\alpha-2, \alpha+T}$, we have

$$
\begin{aligned}
& u(t)=\lambda(F u)(t)<(F u)(t) \\
& \leq|y(u)| \cdot\left|1-\frac{t^{1}}{T+\alpha}\right| \\
& +\frac{t^{\underline{1}}}{\sum_{s=\alpha-2}^{T+\alpha-3} s-\underline{1} g(s)(T+\alpha+\gamma-3-\sigma(s)) \frac{\gamma-1}{-}-(T+\alpha) \Gamma(\gamma)} \mid-y(u) \sum_{s=\alpha-2}^{T+\alpha-3} g(s)(T+\alpha+\gamma-3-\sigma(s)) \frac{\gamma-1}{\left(1-\frac{s^{\underline{1}}}{T+\alpha}\right)}
\end{aligned}
$$

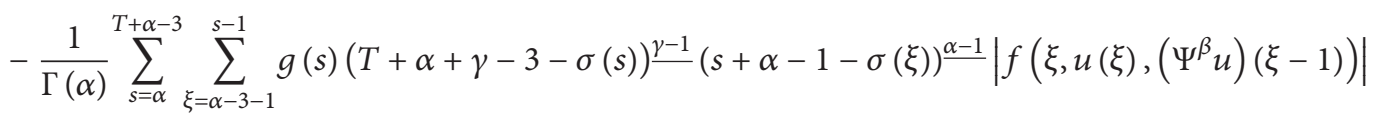

$$
\begin{aligned}
& +\frac{1}{(T+\alpha) \Gamma(\alpha)} \sum_{s=\alpha}^{T+\alpha-3} \sum_{\xi=\alpha-1}^{T+\alpha-1} s^{\underline{1}} g(s)(T+\alpha+\gamma-3-\sigma(s)) \frac{\gamma-1}{(T+2 \alpha-1-\sigma(\xi))^{\alpha-1}}\left|f\left(\xi, u(\xi),\left(\Psi^{\beta} u\right)(\xi-1)\right)\right| \\
& +\frac{t^{\underline{1}}}{(T+\alpha) \Gamma(\alpha)} \sum_{s=\alpha-1}^{T+\alpha-1}(T+2 \alpha-1-\sigma(s))^{\frac{\alpha-1}{}}\left|f\left(s, u(s),\left(\Psi^{\beta} u\right)(s-1)\right)\right|+\frac{1}{\Gamma(\alpha)} \sum_{s=\alpha-1}^{t-1}(t+\alpha-1-\sigma(s))^{\frac{\alpha-1}{}} \\
& \cdot\left|f\left(s, u(s),\left(\Psi^{\beta} u\right)(s-1)\right)\right|<2 L_{2}+L_{1} \frac{2 \Gamma(T+\alpha+1)}{\Gamma(\alpha+1) \Gamma(T+1)}+\left(L_{2} \frac{K[\gamma(T+2)+3] \Gamma(T+\gamma)}{\gamma(\gamma+1) \Gamma(T)}\right. \\
& \left.+L_{1}\left[\frac{K[\gamma(T+2)+3] \Gamma(T+\gamma)}{\gamma(\gamma+1) \Gamma(T)}+\frac{K \Gamma(T+\alpha(\alpha+\gamma)-3) \Gamma(T+\alpha) \Gamma(T+\gamma-2)}{\gamma(\gamma+1) \Gamma(\alpha+1) \Gamma(T-2) \Gamma(T+1)}\right]\right) \cdot\left(\frac{K[T+\alpha-\gamma(2-\alpha)-3]}{\gamma(\gamma+1) \Gamma(T)}\right. \\
& -(T+\alpha) \Gamma(\gamma))^{-1}=L_{2} \Omega+L_{1} \Lambda
\end{aligned}
$$


which implies that, for each $t \in \mathbb{N}_{\alpha-2, \alpha+T}$, we have

$$
\|u\|_{\mathscr{C}} \leq L_{2} \Omega+L_{1} \Lambda
$$

where $\Omega$ and $\Lambda$ are defined on (25). This shows that set $\mathscr{E}$ is bounded. As a consequence of Schaefer's fixed point theorem, we conclude that $F$ has a fixed point which is a solution of problem (1).

\section{Some Examples}

In this section, in order to illustrate our results, we consider some examples.

Example 1. Consider the following fractional sum boundary value problem:

$$
\begin{aligned}
& \Delta^{3 / 2} u(t)=\frac{e^{-(t+1 / 2)}}{5(t+201 / 2)^{2}} \cdot \frac{|u|+1}{1+\sin ^{2} u} \\
& +\sum_{s=-1}^{t-1}\left(t-\frac{1}{2}-\sigma(s)\right)^{\frac{-1 / 2}{2}} u\left(s+\frac{1}{2}\right), \\
& \quad \frac{\arctan \left[\cos ^{2}(t-3 / 2) \pi\right] e^{-2|s-t+7 / 2|}}{2000 \sqrt{\pi}(t+19 / 2)^{2}} \quad t \in \mathbb{N}_{0,4}, \\
& u\left(-\frac{1}{2}\right)=\frac{|u|}{(100 e)^{3}} \sin ^{2}|\pi u|, \\
& u\left(\frac{11}{2}\right)=\Delta^{-11 / 4} u\left(\frac{17}{8}\right)\left[1000 e+200 \cos ^{2}\left(\frac{17}{8}\right)\right] .
\end{aligned}
$$

Here $\alpha=3 / 2, T=4, \beta=1 / 2, y(u)=$ $\left(|u| /(100 e)^{3}\right) \sin ^{2}|\pi u|, \gamma=11 / 4, g(t)=1000 e+200 \cos ^{2} t$, $\varphi(t-1, s+\beta)=e^{-2|s-t+4|} / 2000 \sqrt{\pi}$, and

$$
\begin{aligned}
f\left(t, u(t),\left(\Psi^{\beta} u\right)(t-1)\right) & \\
= & \frac{e^{-t}}{5(t+100)^{2}} \cdot \frac{|u|+1}{1+\sin ^{2} u} \\
& +\left[\frac{\arctan \left[\cos ^{2}(t-2) \pi\right]}{(t+9)^{2}}\right]\left[\Delta^{-1 / 2} \varphi u\right]\left(t+\frac{1}{2}\right) .
\end{aligned}
$$

Let $t \in \mathbb{N}_{1 / 2,9 / 2}$; we have

$$
\begin{aligned}
& \mid f\left(t, u(t),\left(\Psi^{1 / 2} u\right)(t-1)\right) \\
& \quad-f\left(t, v(t),\left(\Psi^{1 / 2} u\right)(t-1)\right)\left|\leq \frac{4}{404010}\right| u-v \mid \\
& \quad+\frac{22}{2527}\left|\left(\Psi^{1 / 2} u\right)-\left(\Psi^{1 / 2} v\right)\right|,
\end{aligned}
$$

so $\left(H_{1}\right)$ holds with $\tau_{1}=4 / 404010, \tau_{2}=22 / 2527$, and we have $\varphi_{0}=1 / 2000 e^{8} \sqrt{\pi}$, and

$$
\begin{aligned}
& |y(u)-y(v)| \\
& \quad=\left|\frac{|u|}{(100 e)^{3}} \sin ^{2}\right| \pi u\left|-\frac{|v|}{(100 e)^{3}} \sin ^{2}\right| \pi v|| \\
& \quad \leq \frac{1}{(100 e)^{3}}\|u-v\|_{\mathscr{C}},
\end{aligned}
$$

so $\left(H_{2}\right)$ holds with $\mu=1 /(100 e)^{3}$.

Since $1000 e \leq g(t) \leq 1000 e+200=K$, we have

$$
\begin{aligned}
& K[T+\alpha-\gamma(2-\alpha)-3]-(T+\alpha) \Gamma(\gamma+2) \Gamma(T) \\
& \quad \approx 547.011>0 ;
\end{aligned}
$$

then $\left(\mathrm{H}_{3}\right)$ is satisfied.

Also, we have

$$
\begin{aligned}
& \Omega \approx 47128.501, \\
& \Lambda \approx 14297.052 .
\end{aligned}
$$

We can show that

$$
\begin{aligned}
& \mu \Omega+\left(\tau_{1}+\tau_{2} \frac{\varphi_{0}(T+\beta+2)^{\frac{\beta}{-}}}{\Gamma(\beta+1)}\right) \\
& \quad \cdot\left[\Lambda+\frac{2 \Gamma(T+\alpha+1)}{\Gamma(\alpha+1) \Gamma(T+1)}\right] \\
& =\frac{1}{(100 e)^{3}}(47128.501)+(14297.052) \\
& \quad \cdot\left[\frac{4}{404010}+\left(\frac{22}{2527}\right) \frac{(13 / 2) \frac{1 / 2}{2000 e^{8} \sqrt{\pi} \Gamma(3 / 2)}}{20.144}\right] \approx 0.1
\end{aligned}
$$

Hence, by Theorem 7, boundary value problem (33) has a unique solution.

Example 2. Consider the following fractional sum boundary value problem:

$$
\begin{aligned}
& \Delta^{3 / 2} u(t)=\frac{(t+1 / 2)^{1 / 2} e^{-3(t+1 / 2)}}{1+(t+1 / 2)\left|1+\cos ^{2}(u+\pi)\right|} \\
& +\sum_{s=-1}^{t-1}\left(t-\frac{1}{2}-\sigma(s)\right)^{\frac{-1 / 2}{}} \\
& \quad \cdot \frac{(t+3 / 2)(s+1 / 2) e^{-(s+1 / 2)^{2}}}{\sqrt{\pi}|(t+3 / 2)(s+1 / 2)-(t+1 / 2)|} u\left(s+\frac{1}{2}\right),
\end{aligned}
$$

$$
t \in \mathbb{N}_{0,3} \text {, }
$$

$$
\begin{aligned}
& u\left(-\frac{1}{2}\right)=\frac{\left|u^{2}+2\right| \frac{1-\left|u^{2}+2\right|}{\pi+u^{2}}}{u\left(\frac{9}{2}\right)=\Delta^{-8 / 3} u\left(\frac{11}{6}\right)\left(12 e+\sin \left(\frac{11}{6}\right)\right)^{2} .}
\end{aligned}
$$


Here $\alpha=3 / 2, T=3, \beta=1 / 2, y(u)=\left|u^{2}+2\right| \frac{1-\left|u^{2}+2\right|}{\mid} /(\pi+$ $\left.u^{2}\right), \gamma=8 / 3$ and $g(t)=(12 e+\sin t)^{2}, \varphi(t-1, s+\beta)=(t+$ $1)(s+1 / 2) e^{-(s+1 / 2)^{2}} /|(t+1)(s+1 / 2)-t|$, and

$$
\begin{aligned}
& f\left(t, u(t),\left(\Psi^{\beta} u\right)(t-1)\right)=\frac{\sqrt{t} e^{-3 t}}{1+t\left|1+\cos ^{2}(u+\pi)\right|} \\
& \quad+\sum_{s=-1}^{t-3 / 2}(t-1-\sigma(s)) \frac{-1 / 2}{} \varphi\left(t-1, s+\frac{1}{2}\right) \\
& \cdot u\left(s+\frac{1}{2}\right) .
\end{aligned}
$$

Clearly for $t \in \mathbb{N}_{1 / 2,7 / 2}$, we have

$$
\varphi_{0}<\frac{|(t+1)(s+1 / 2)-t|+t}{|(t+1)(s+1 / 2)-t|}<1+\frac{1}{|(s+1 / 2)-1|}
$$$$
\leq 3
$$

$$
\begin{aligned}
& \left|f\left(t, u(t),\left(\Psi^{1 / 2} u\right)(t)\right)\right| \leq\left|\frac{\sqrt{t}}{1+t}+\frac{\varphi_{0}(9 / 2) \frac{-1 / 2}{\Gamma(3 / 2)}}{\quad}\right| \\
& \quad<2.566=L_{1},
\end{aligned}
$$

$$
\begin{aligned}
& |y(u)|=\frac{\Gamma\left(\left|u^{2}+2\right|+1\right)}{\left(\pi+u^{2}\right) \Gamma\left(2\left|u^{2}+2\right|\right)}<\frac{1}{\pi}=L_{2}, \\
& (12 e)^{2} \leq g(t) \leq(12 e+1)^{2}=K, \\
& \frac{1}{6}(12 e+1)^{2}-\frac{9}{2} \Gamma\left(\frac{14}{3}\right) \Gamma(3)=55.974>0 .
\end{aligned}
$$

Hence, conditions $\left(H_{3}\right),\left(H_{5}\right)$, and $\left(H_{6}\right)$ of Theorem 11 are satisfied, and consequently boundary value problem (40) has at least one solution.

\section{Conflict of Interests}

The authors declare that there is no conflict of interests regarding the publication of this paper.

\section{Acknowledgments}

The authors would like to thank the editor and the referees for their useful comments. This research was funded by King Mongkut's University of Technology North Bangkok (Contract no. KMUTNB-GOV-58-50).

\section{References}

[1] G.-C. Wu and D. Baleanu, "Discrete fractional logistic map and its chaos," Nonlinear Dynamics, vol. 75, no. 1-2, pp. 283-287, 2014.

[2] G.-C. Wu and D. Baleanu, "Chaos synchronization of the discrete fractional logistic map," Signal Processing, vol. 102, pp. 96-99, 2014.

[3] R. P. Agarwal, D. Baleanu, S. Rezapour, and S. Salehi, "The existence of solutions for some fractional finite difference equations via sum boundary conditions," Advances in Difference Equations, vol. 2014, article 282, 2014.
[4] W. Lv, "Existence of solutions for discrete fractional boundary value problems with a $p$-Laplacian operator," Advances in Difference Equations, vol. 2012, article 163, 10 pages, 2012.

[5] S. Kang, Y. Li, and H. Chen, "Positive solutions to boundary value problems of fractional difference equation with nonlocal conditions," Advances in Difference Equations, vol. 2014, article 7, 2014.

[6] C. S. Goodrich, "On a discrete fractional three-point boundary value problem," Journal of Difference Equations and Applications, vol. 18, no. 3, pp. 397-415, 2012.

[7] C. S. Goodrich, "Existence and uniqueness of solutions to a fractional difference equation with nonlocal conditions," Computers and Mathematics with Applications, vol. 61, no. 2, pp. 191-202, 2011.

[8] R. A. C. Ferreira, "Existence and uniqueness of solution to some discrete fractional boundary value problems of order less than one," Journal of Difference Equations and Applications, vol. 19, no. 5, pp. 712-718, 2013.

[9] T. Abdeljawad, "On Riemann and Caputo fractional differences," Computers and Mathematics with Applications, vol. 62, no. 3, pp. 1602-1611, 2011.

[10] T. Abdeljawad, "Dual identities in fractional difference calculus within Riemann," Advances in Difference Equations, vol. 2013, article 36, 2013.

[11] T. Abdeljawad, "On delta and nabla Caputo fractional differences and dual identities," Discrete Dynamics in Nature and Society, vol. 2013, Article ID 406910, 12 pages, 2013.

[12] F. M. Atici and P. W. Eloe, "Two-point boundary value problems for finite fractional difference equations," Journal of Difference Equations and Applications, vol. 17, no. 4, pp. 445-456, 2011.

[13] F. M. Atici and P. W. Eloe, "A transform method in discrete fractional calculus," International Journal of Difference Equations, vol. 2, no. 2, pp. 165-176, 2007.

[14] T. Sitthiwirattham, J. Tariboon, and S. K. Ntouyas, "Existence results for fractional difference equations with three-point fractional sum boundary conditions," Discrete Dynamics in Nature and Society, vol. 2013, Article ID 104276, 9 pages, 2013.

[15] T. Sitthiwirattham, J. Tariboon, and S. K. Ntouyas, "Boundary value problems for fractional difference equations with threepoint fractional sum boundary conditions," Advances in Difference Equations, vol. 2013, article 296, 2013.

[16] T. Sitthiwirattham, "Existence and uniqueness of solutions of sequential nonlinear fractional difference equations with three-point fractional sum boundary conditions," Mathematical Methods in the Applied Sciences, vol. 38, pp. 2809-2815, 2015.

[17] T. Sitthiwirattham, "Boundary value problem for $p$-Laplacian Caputo fractional difference equations with fractional sum boundary conditions," Mathematical Methods in the Applied Sciences, 2015.

[18] D. H. Griffel, Applied Functional Analysis, Ellis Horwood, Chichester, UK, 1981.

[19] H. Schaefer, "Über die Methode der a priori-Schranken," Mathematische Annalen, vol. 129, no. 1, pp. 415-416, 1955. 


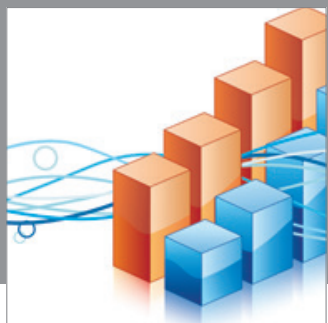

Advances in

Operations Research

mansans

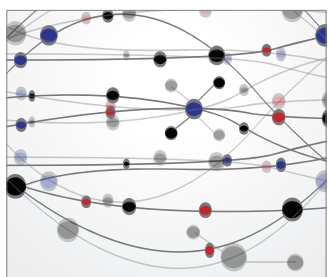

The Scientific World Journal
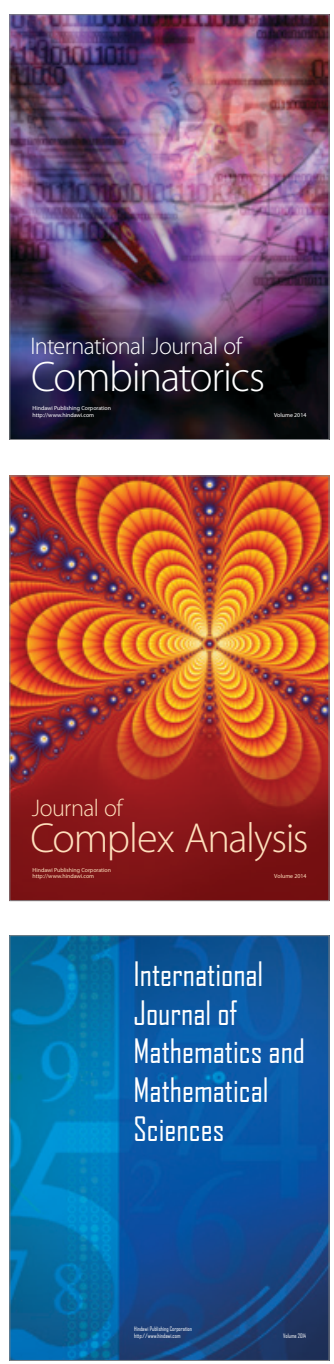
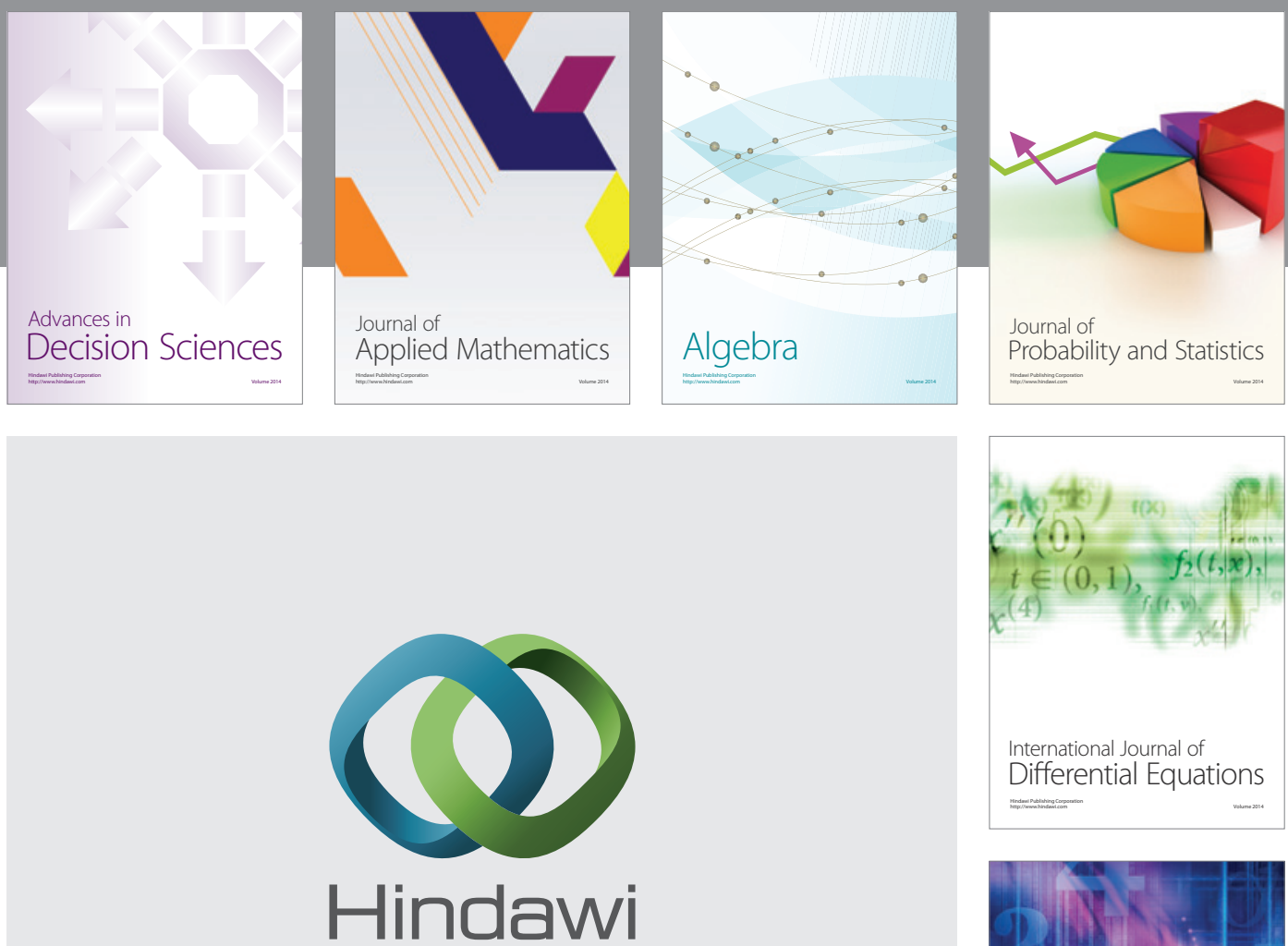

Submit your manuscripts at http://www.hindawi.com
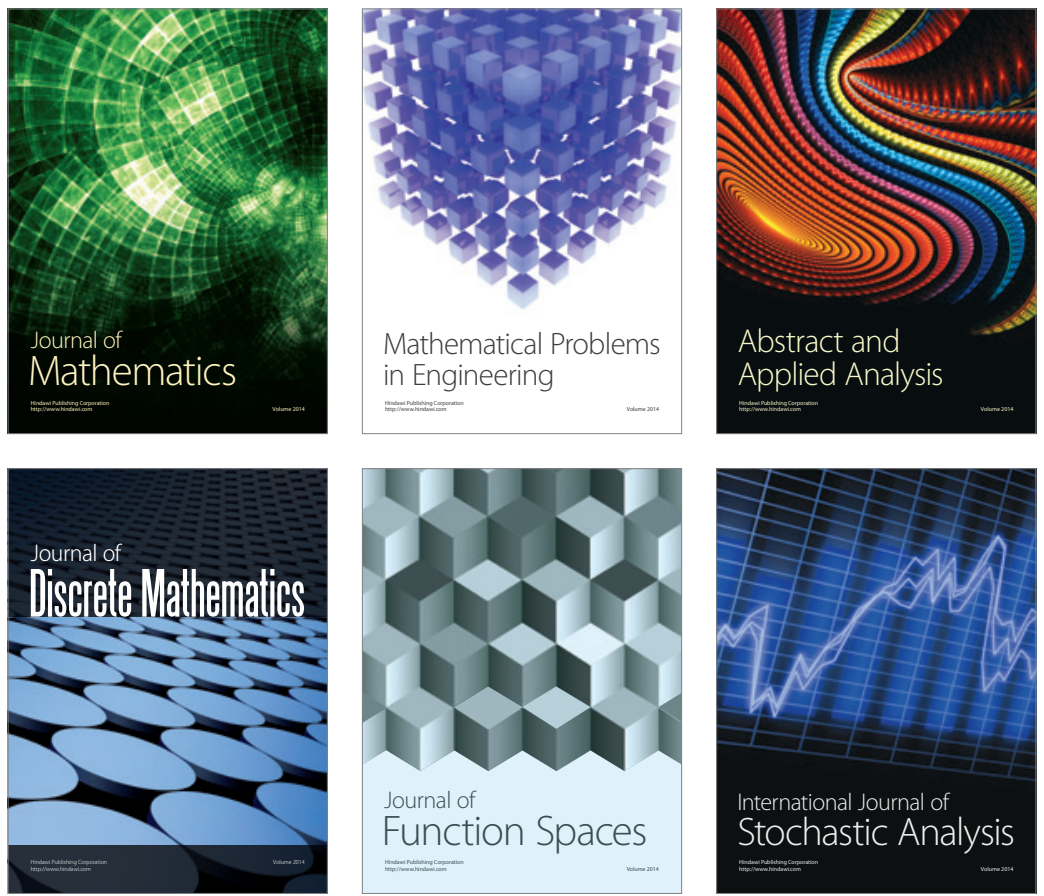

Journal of

Function Spaces

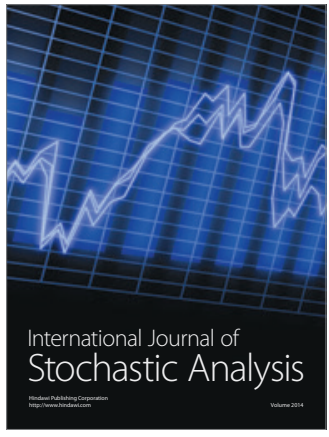

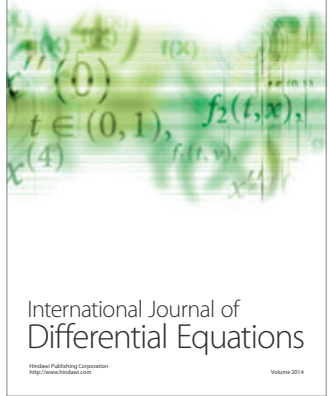
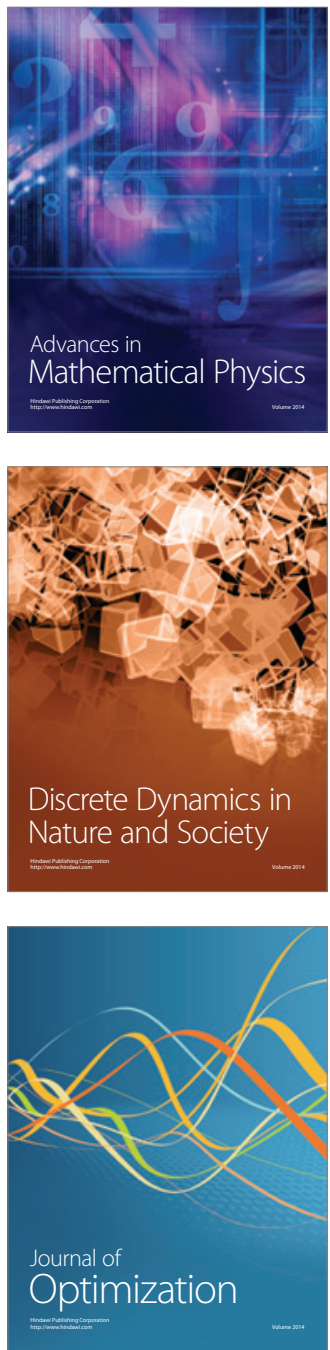